\title{
Do guidelines improve the diagnosis and investigation of hyponatraemia?
}

Dr Chloe Broughton, Dr Emily Lucas, Dr Beas Bhattacharya

\section{Background}

- Hyponatraemia is common and associated with significant morbidity and mortality. However, it's often not recognised as a serious diagnosis and therefore inadequately investigated and poorly managed.

- An audit of the management of patients admitted to The Great Western Hospital (GWH) with hyponatraemia confirmed these problems. A hyponatraemia guideline was produced in order to improve diagnosis, investigation and management of patients with hyponatraemia.

\section{Methods}

- The guideline was piloted in the Acute Medical Unit at GWH.

- Following a teaching session and introductory period, a re-audit was performed. Data was collected retrospectively in patients with an admission sodium of $\leq 127 \mathrm{mmol} / \mathrm{L}$, over a one month period.

- The aim was to identify whether introduction of a guideline improved diagnosis, investigation and management of patients.

\section{Results}

- 20 patients: 8 male $\& 12$ female

Mean age 64 years (range 25 - 88 years)
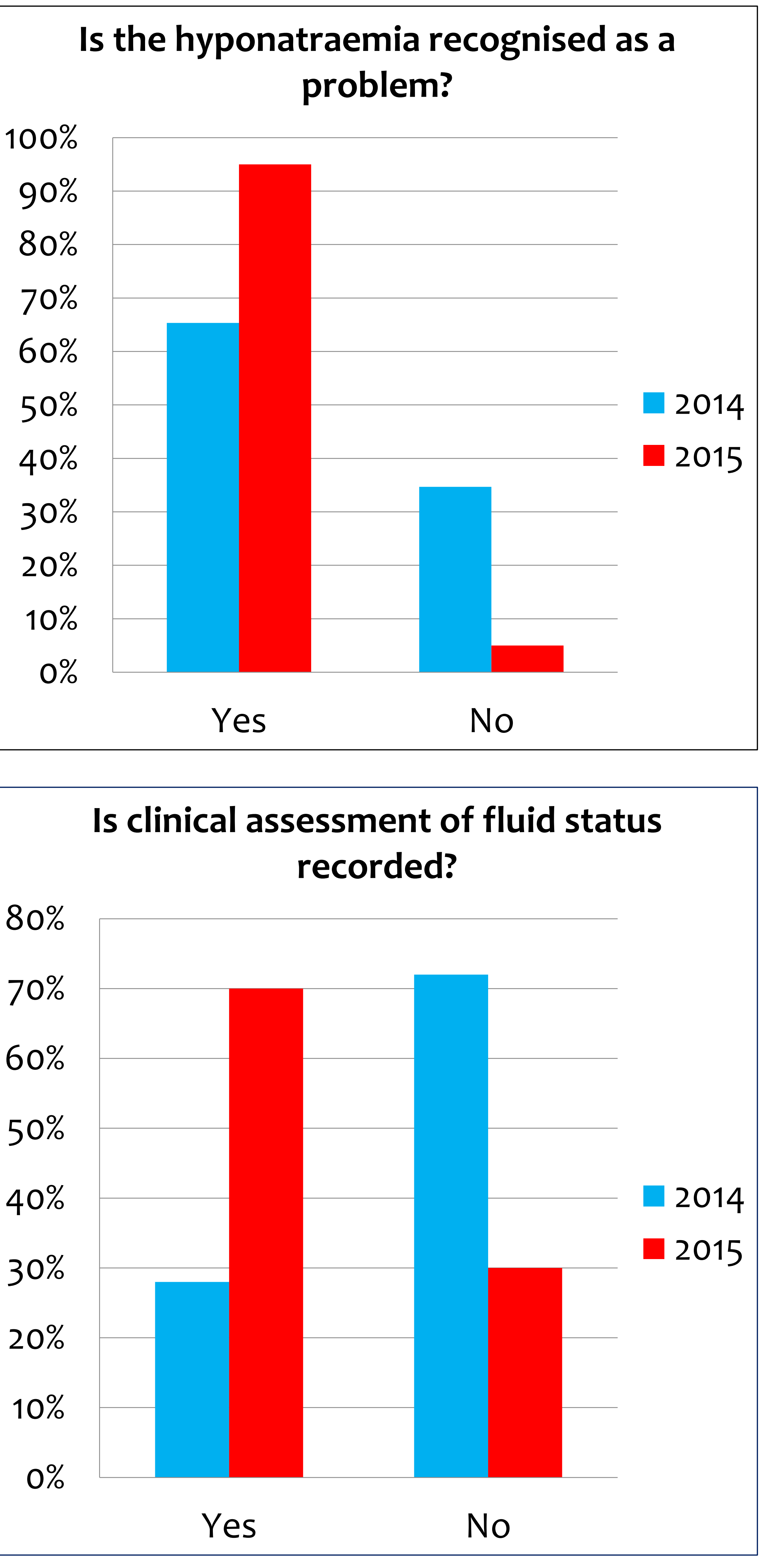
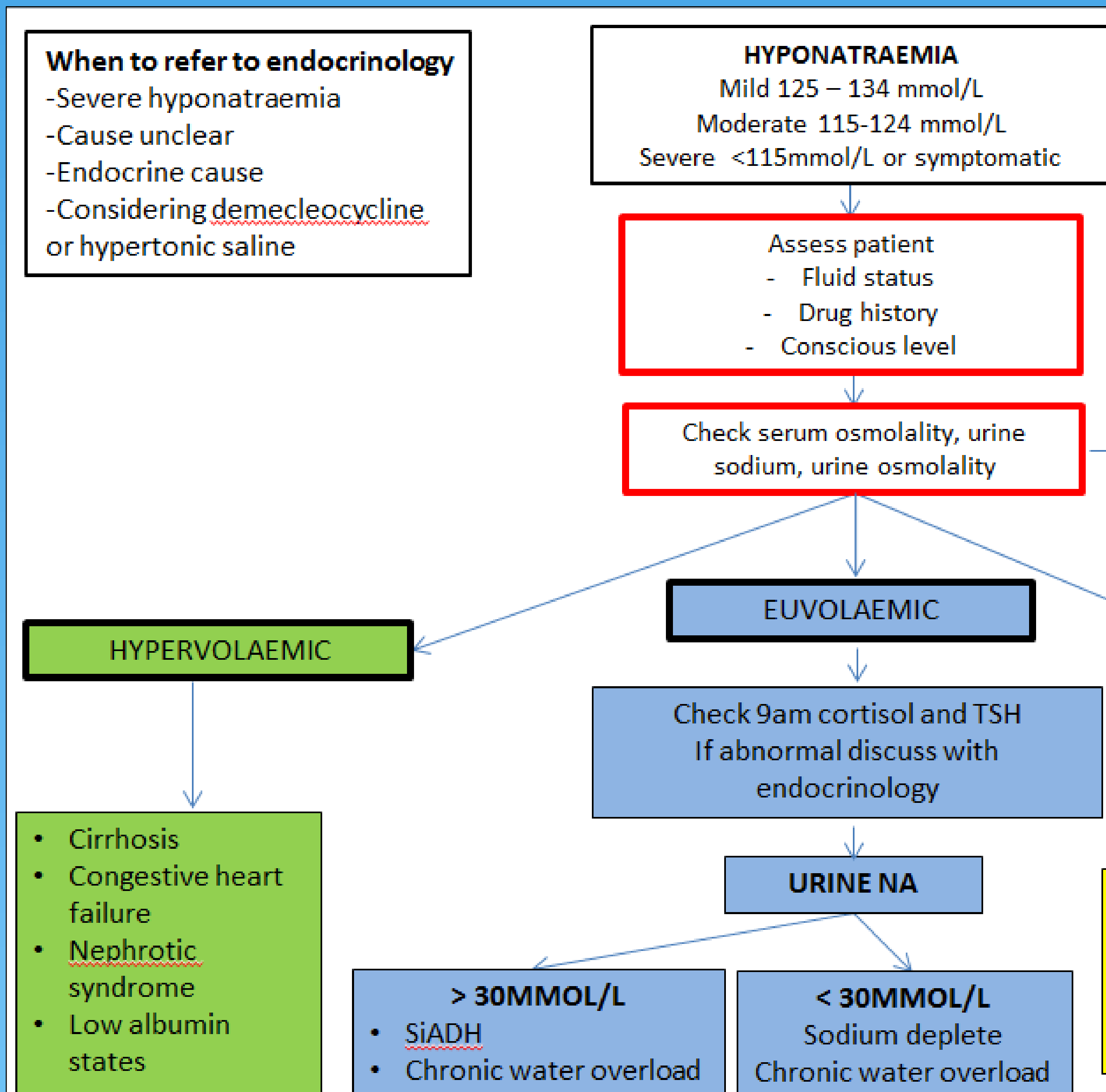

endocrinology
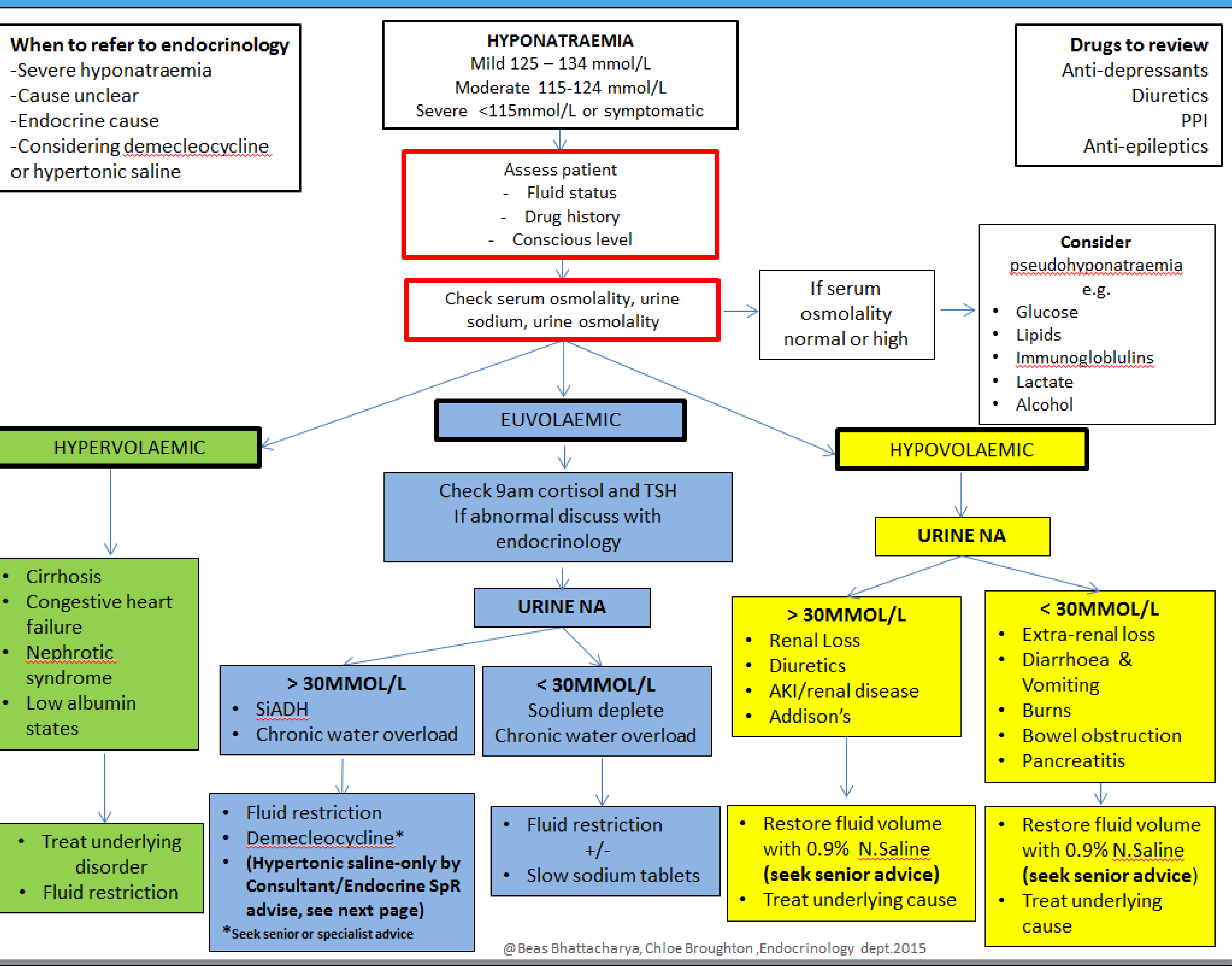

- Treat underlying cause

cause

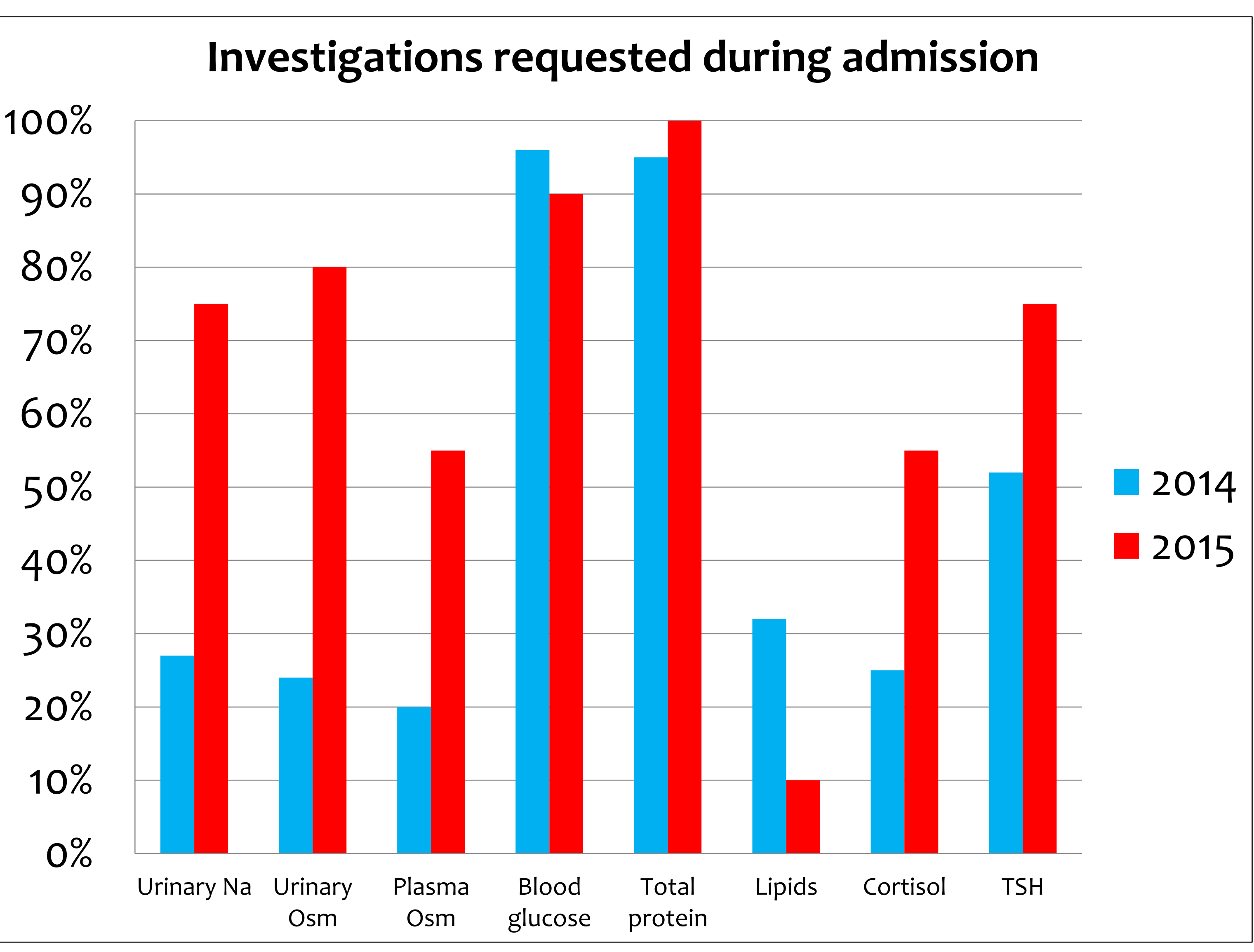

Percentage of patients referred to Endocrinology Team

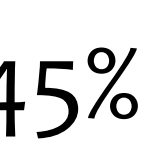

$40 \%$

$35 \%$

$30 \%$

$25 \%$

$20 \%$

$15 \%$

$10 \%$

$5 \%$

$0 \%$

\section{Conclusion}

- This audit demonstrates that the use of education and a guideline has improved recognition of hyponatraemia.

- It has resulted in an increase in the number of relevant investigations requested and in the number of patients reviewed by the endocrine team. 